NASA Contractor Report 3862

\title{
On the Long Range Propagation of Sound Over Irregular Terrain
}

M. S. Howe

CONTRACT NAS1-16521

DECEMBER 1984 

NASA Contractor Report 3862

\section{On the Long Range Propagation of Sound Over Irregular Terrain}

M. S. Howe

Bolt Beranek and Newman Inc.

Cambridge, Massachusetts

Prepared for

Langley Research Center

under Contract NAS1-16521

\section{N/SA}

National Aeronautics

and Space Administration

Scientific and Technical

Information Branch 
TABLE OF CONTENTS

\section{Page}

SUMMARY...............................

LIST OF PRINCIPAL SYMBOLS.................... Vii

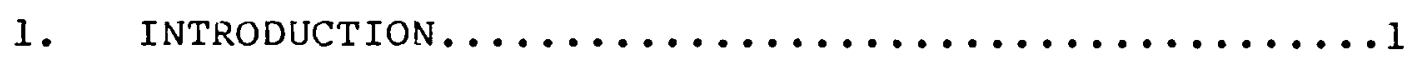

2. COHERENT ACOUSTIC BOUNDARY CONDITION AT A

ROUGH SURFACE OF FINITE NORMAL IMPEDANCE........5

3. LONG RANGE PROPAGATIUN FROM A POINT SOURCE

CLOSE TO THE GROUND: TWO DIMENSIONAL

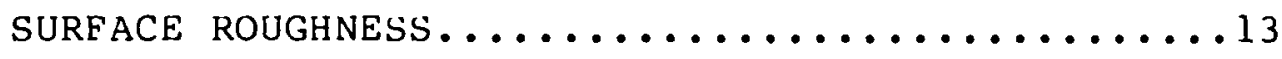

3.1 Propagation Over Randomly Irregular Terrain.....17

3.2 One-Dimensional Surface Irregularities.......... 8

4. CONCLUSION............................. 19

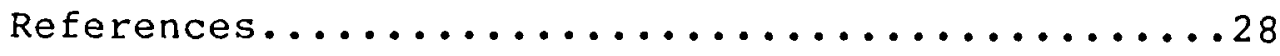





\section{SUMMARY}

The theory of sound propagation over randomly irregular, nominally plane terrain of finite impedance is discussed. The analysis is an extension of the theory of coherent scatter originally proposed by Biot [28] for an irregular rigid surface. It combines Biot's approach, wherein the surface irregularities are modeled by a homogeneous distribution of hemispherical "bosses," with more conventional analyses in which the ground is modeled as a smooth plane of finite impedance. At sufficiently low frequencies it is shown that the interaction of the surface irregularities with the nearfield of a ground based source leads to the production of "surface waves," which are effective in penetrating the ground shadow zone predicted for a smooth surface of the same impedance. 


\section{LIST OP PRINCIPAL SYMBOLS}

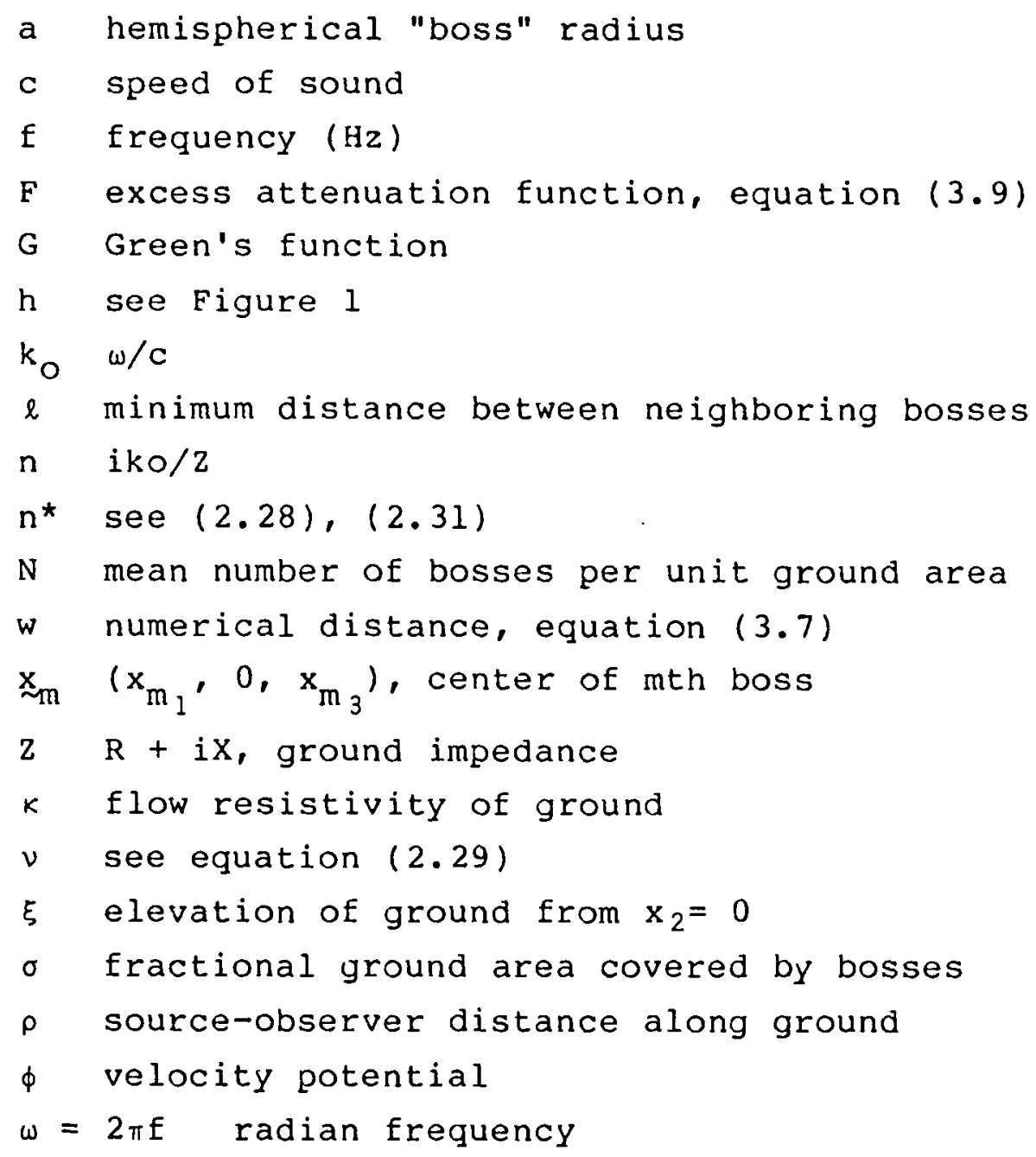




\section{INTRODUCTION}

A plane sound wave is incident on a flat surface of finite impedance. As the angle of incidence approaches grazing the amplitude of the reflected wave tends to that of the incident wave, but their phases ultimately differ by $180^{\circ}$ [1]. Thus a plane acoustic wave cannot propagate parallel to the surface, and, in particular, this implies that most of the surface would be in a "shadow zone" of an acoustic source located on or very close to the surface. The intensity of the sound in this shadow zone is very much less than it would be at the same distance from the source had the surface been an ideal, rigid reflector. The point source problem has been studied extensively in the literature (see, e.g., the reviews [2-4]), and is the acoustic analogue of the problem of radiation from an electromagnetic dipole situated above a conducting boundary [5-7]. Detailed analyses for an acoustic point source are given in references [817 .

These studies indicate that the shadow zone is penetrated by a "ground wave" whose properties depend on the surface impedance characteristics. Under certain conditions the ground wave includes a trapped surface wave which propagates subsonically in the acoustic medium just above the surface. The amplitude of this wave decays inversely as the square root of the propagation distance $r$, say, due to cylindrical spreading from the source, and exponentially with $r$ due to absorption by the surface. If the absorption is sufficiently small, however, there can be a considerable interval in $r$ within the shadow zone wherein the acoustic field exceeds that in free space at the same distance from the source (i.e., the surface wave provides a temporary means of negative damping). 
There is confusion and controversy in the literature regarding the role of the surface wave component of the ground wave $[14,16]$. This has arisen because account has not always been taken of changes in the asymptotic formula for the ground wave function $F(w)$ in different regions of the $w-p l a n e, w$ being the complex numerical distance (see sec. 3). Accordingly, experimental studies often involve conflicting interpretations of the measured sound pressure levels (see e.g., refs $[2,3,18-22]$ ).

The surface wave phenomenon has recently been discussed by Tolstoy [23-27] in connection with the propagation of sound over a randomly irregular surface. This work is a development of an earlier treatment of the problem due to Biot [28-30], involving propagation over a rigid surface formed by a homogeneous distribution of hemispherical bosses on a plane. The surface diffracted sound comprises a coherent component (obtained by averaging with respect to an ensemble of realizations of the distribution of bosses) and an incoherent component (having zero mean). When the acoustic wavelength is large relative to the spacing of the bosses, the incoherent field is small, and may be neglected. In these circumstances the coherent radiation is found to satisfy an impedance condition on a mean, smoothed plane, an important property of which is that it leads to the prediction of a coherent surface wave. Since the composite irregular surface is rigid, however, this wave is not attenuated by absorption at the boundary, although it must ultimately decay due to scattering into incoherent waves. At low Erequencies the influence of such scattering is important only over very large propagation distances. In consequence there is effectively no surface shadow zone; on the contrary the surface pressure tends to be enhanced. Tolstoy [24] has demonstrated theoretically that the surface wave can also eliminate shadowing caused by a vertically decreasing sound speed profile (which tends to refract waves away from the surface). 
Experiments performed by Medwin et al [31], using an acoustically rigid irregular surface, confirm the presence of the surface wave, and are in excellent detailed agreement with the coherent wave theory. It is possible that this effect will also be significant for propagation over homogeneous irregular ground cover of finite impedance, and that there may exist ranges in frequency and propagation distance within which the surface wave will provide a degree of negative damping.

In this paper the Biot/Tolstoy theory is extended to examine the interaction of sound with an irregular surface of finite normal impedance. Attention is given, in particular, to the problem of long range propagation over irregular terrain when both the source and observer are on or close to the ground, and when, in addition, the relevant acoustic wavelengths are large relative to the scale of the surface irregularities. The latter approximation is necessary to make the analysis tractable, but may otherwise be justified on the basis that sound whose wavelength is comparable to, or smaller than, the scale of the surface irregularities, will be rapidly dissipated by surface scattering. Indeed, according to Piercy et al [2], for broadband noise sources and propagation distances of a mile or more, the high frequencies are rapidly attenuated by atmospheric absorption, midfrequencies by ground shadowing, and the main contribution to the measured sound pressure level is from surface waves of frequencies $\mathrm{f}<200 \mathrm{~Hz}$.

In Section 2 of this paper the Biot/Tolstoy boundary condition for the coherent field is determined for an irregular, locally reacting surface. The analysis is restricted to one-and two-dimensional surface roughness elements modeled respectively by a distribution of parallel cylinders of semi-circular crosssection, and hemispherical bosses. There is, of course, no necessity to limit consideration to such specific roughness 
shapes, but more general formulations of the theory (c.f. [23]) are unlikely to alter the principal conclusions of this paper, provided the distribution of surface irregularities is sufficiently homogeneous. The boundary condition is used to study propagation from a ground-sited point source to an observer also positioned on the ground ( $\mathrm{Sec}, 3$ ), making use of empirical estimates of the ground normal impedance. In particular the influence of surface roughness on the ground shadow zone is examined. 


\section{COHERENT ACOUSTIC BOUNDARY CONDITION AT A ROUGH SURFACE OF FINITE NORMAL IMPEDANCE}

Consider the propagation of sound in the region $x_{2}>\zeta\left(x_{\alpha}\right) \quad(\alpha=1,3)$ of a rectangular coordinate system $x_{1}, x_{2}, x_{3}$, where $x_{2}=\zeta\left(x_{\alpha}\right)$ is the rough surface formed by a distribution of hemispherical bosses ( $N$ per unit area) of radius a over the plane $x_{2}=0$ (see Figure 1 ). The length $\ell \sim 1 / \sqrt{\mathrm{N}}$ is taken to characterize the spacing of the bosses, and it is assumed that $\ell \gg>a$. The sound has time harmonic dependence $e^{-i \omega t}$ (which is suppressed in the following), and satisfies the Helmholtz equation

$$
\left(\nabla^{2}+k_{o}^{2}\right) \phi=0, x_{2}>\zeta\left(x_{\alpha}\right),
$$

$\phi$ being the velocity potential, $k_{0}=\omega / c>0$, and $c$ is the speed of sound. Greek suffixes, $\alpha, \beta$, etc, range over the 1,3 directions only.

On the irregular surface the potential satisfies

$$
\frac{\partial \phi}{\partial x_{n}}+n \phi=0, x_{2}=\zeta\left(x_{\alpha}\right),
$$

where $x_{n}$ is a local coordinate normal to the surface and directed into the acoustic medium, and where

$$
\mathrm{n}=i \mathrm{k}_{\mathrm{o}} / \mathrm{Z}
$$

and

$$
z=R+i x
$$

is the specific normal impedance, assumed to depend on frequency alone. Attention is confined to sound of sufficiently low frequency that 


$$
\frac{1}{|2|} \sim \mathrm{k}_{0} \mathrm{a} \ll 1 .
$$

The magnitude of $Z$ in terrestrial applications is discussed in Section 3.

Introduce a control surface $S: x_{2}=h(h>a)$, just above the irregular boundary, and consider the diffraction of an incident wave denoted by $\phi_{\mathrm{I}}$. In $\mathrm{x}_{2}>\mathrm{h}$ the total field can be expressed in the form

$$
\phi(\underset{\sim}{x})=\phi_{I}(\underset{\sim}{x})+\phi_{R}(\underset{\sim}{x})+\oint_{S} \frac{\partial \phi}{\partial Y_{2}}(y) G(\underset{\sim}{x}, \chi) d y_{1} d y_{3},
$$

the surface integral being taken over $S$. In $(2.6) \phi_{R}(x)$ is the wave that would be specularly reflected from a rigid plane coinciding with $S$, and the Green's function $G(\underset{\sim}{x}, y)$ has outgoing wave behavior in $x_{2}>h$, satisfies $\partial G / \partial y_{2}=0$ on $S$, and is given by

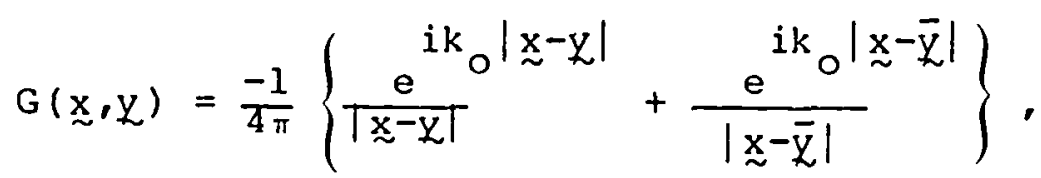

where $\bar{Z}=\left(y_{1},-y_{2}+2 h, y_{3}\right)$.

We now derive the leading order approximation to the coherent (average) form of $(2.6)$.

The integration in (2.6) may be expressed as the sum of integrals over a sequence of non-overlapping area elements $s_{m}$ of s. Each $S_{m}$ covers precisely one hemispherical boss, and has a diameter of the same order as the separation distance $\ell$. Let $x_{m} \equiv\left(x_{m_{1}}, 0, x_{m_{3}}\right)$ denote the position of the center of the boss in $S_{m}$. In the neighborhood of $x_{m}$ the coherent field $\bar{\phi}(x)$, say, can be expressed in the form 


$$
\begin{aligned}
\bar{\phi}(\underset{\sim}{x}) & =\bar{\phi}\left(x_{m}^{x}\right)+\left(x_{j}-x_{m j}\right) \frac{\partial \bar{\phi}}{\partial x_{j}}\left({\underset{\sim}{m}}_{m}\right) \\
& +\frac{1}{2}\left(x_{i}-x_{m i}\right)\left(x_{j}-x_{m j}\right) \frac{\partial 2 \bar{\phi}}{\partial x_{i} \partial x_{j}}\left(x_{m}\right)+\ldots
\end{aligned}
$$

(no summation with respect to the repeated suffix $m$ ). It follows from (2.2) - (2.5) that

$$
\frac{\partial \bar{\phi}}{\partial x_{\alpha}} \sim k_{0} \bar{\phi} ; \quad \frac{\partial \bar{\phi}}{\partial x_{2}} \sim k_{0}^{2} a \bar{\phi},
$$

and that the final term on the right of $(2.8) \sim 0\left(k_{0} l\right)^{2} \bar{\phi}$, at most.

This ordering suggests the following representation of $\phi(x)$ in $s_{m}$ :

$$
\phi(\underset{\sim}{x})=\bar{\phi}\left({\underset{\sim}{m}}_{m}\right)+\phi_{1}(\underset{\sim}{x})+\phi_{2}(\underset{\sim}{x})+\ldots
$$

where

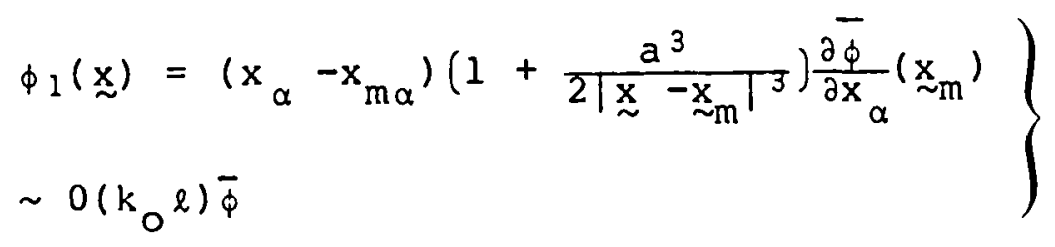

and $\phi_{2}$ contains all remaining terms $\sim 0\left(k_{0} l\right)^{2 \bar{\phi}}$. Observe that, correct to $0\left(k_{0} l\right)^{2}$, this representation implies that

$$
\nabla^{2} \phi_{1}=0 ; \nabla^{2} \phi_{2}=-k_{0}^{2} \bar{\phi}\left({\underset{\sim}{m}}_{m}\right),
$$

and that $\partial \phi_{1} / \partial x_{n}=0$ on the surface $S_{m o}$ comprising the portion of the plane $x_{2}=0$ and hemispherical boss in $S_{m}$ (see Figure 1 ). Hence, to the same approximation, (2.2) becomes in $s_{m}$ : 


$$
\frac{\partial \phi_{2}}{\partial x_{n}}=-n \bar{\phi}\left(x_{m \alpha}\right), x_{2}=\zeta\left(x_{\alpha}\right)
$$

Next, for an observer at $x$ at a large distance from $X$ in $s_{m,}(2.7)$ becomes

$$
G(\underset{\sim}{x}, \mathcal{L})=G\left(\underset{\sim}{x},{\underset{\sim m}{m}}^{x}\right)+\left(y_{\alpha}-x_{m \alpha}\right) \frac{\partial G}{\partial x_{m \alpha}}\left(\underset{\sim}{x},{\underset{\sim m}{m}}^{x}\right)+\ldots
$$

where each term in this expansion is $0\left(k_{0} \ell\right)$ relative to the preceeding one.

The expansions (2.10), (2.14) are now used to determine the principal contribution from $s_{m}$ to the integral in (2.6). Consider first that arising from $\phi_{1}(\underset{\sim}{x})$. Since $\ell>>a$, one finds, correct to the retention of terms of order $\left(k_{0} a\right)^{2}$,

$$
\begin{aligned}
& \oint_{S_{m}} \frac{\partial \phi_{1}}{\partial y_{2}}(X) G(\underset{\sim}{x}, Z) d Y_{1} d y_{3} \\
& =\oint_{S_{m}}\left(y_{\alpha}-x_{m \alpha}\right) \frac{\partial \phi_{1}}{\partial y_{2}}(y) \frac{\partial G}{\partial x_{m}}\left(\underset{\sim}{x},{\underset{m}{m}}_{m}\right) d y_{1} d y_{3} \\
& =-\pi a^{3} \frac{\partial \bar{\phi}}{\partial x_{m}}\left(x_{m}\right) \frac{\partial G}{\partial x_{m \alpha}}\left(\underset{\sim}{x}, x_{m}\right),
\end{aligned}
$$

To the same order of approximation, the net coherent contribution to the surface integral in (2.6) Erom all terms of this type can be set in the form

$$
\underset{m}{\sum} \oint_{m} \frac{\partial \phi_{1}}{\partial y_{2}} G(\underset{\sim}{x}, \mathcal{L}) d y_{1} d y_{3}=\oint_{S} \sigma a \frac{\partial 2 \bar{\phi}}{\partial y_{\alpha}^{2}} G(\underset{\sim}{x}, \mathcal{L}) d y_{1} d y_{3},
$$

where

$$
\sigma=N \pi a^{2}
$$


is the mean fractional surface area of the plane $x_{2}=0$ covered by the hemispheres.

Similarly, making use of the divergence theorem, one obtains

$$
\begin{aligned}
& \underset{S_{m}}{\oint} \frac{\partial \phi_{2}}{\partial Y_{2}}(\chi) G(\underset{\sim}{x}, \chi) d y_{1} d y_{3} \approx \oint_{S_{m}}^{\oint} \frac{\partial \phi_{2}}{\partial y_{2}}(\chi) G\left(\underset{\sim}{x}, \underset{\sim m}{x_{m}}\right) d y_{1} d y_{3}
\end{aligned}
$$

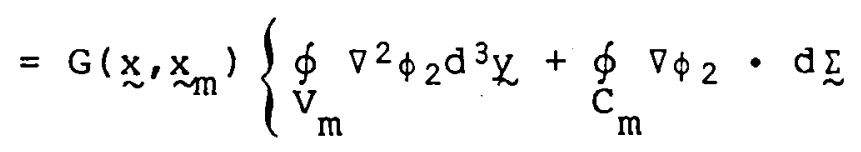

$$
\begin{aligned}
& \left.+\oint_{\mathrm{mo}} \nabla \phi_{2} \cdot \mathrm{d} \Sigma \underset{\sim}{\}}\right\}
\end{aligned}
$$

where $v_{m}$ is the volumetric region bounded by $s_{m}$, $s_{m o}$ (see Figure $1)$ and laterally by the surface $c_{m}$, and $d \Sigma$ is the surface element directed into $\mathrm{V}_{\mathrm{m}}$.

The individual coherent components of the integrals in the brace brackets of $(2.18)$ are evaluated as follows:

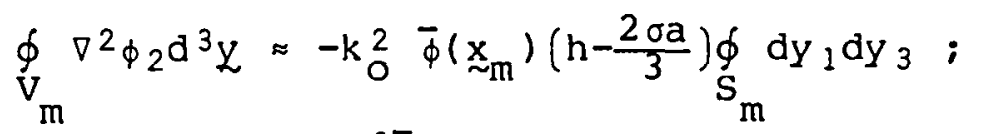

$$
\begin{aligned}
& \underset{C_{m}}{\oint} \nabla \phi_{2} \cdot d \Sigma=\frac{-\partial^{2} \bar{\phi}}{\partial x_{\alpha}^{2}}\left(x_{m}\right) h \underset{S_{m}}{\phi} d y_{1} d y_{3} ; \\
& \oint_{\mathrm{S}_{\mathrm{mo}}} \nabla \phi_{2} \cdot d \sum_{\sim} \approx-n(1+\sigma) \bar{\phi}\left(\mathrm{x}_{\mathrm{m}}\right) \underset{\mathrm{S}_{\mathrm{m}}}{\oint_{1}} \mathrm{dy} \mathrm{y}_{1} \mathrm{~d} \mathrm{y}_{3} \cdot
\end{aligned}
$$

In obtaining these results use has been made of (2.12) and (2.13), and it has been noted that on $c_{m}, \phi_{2}$ may be replaced by

$$
\begin{aligned}
\bar{\phi}_{2}(\underset{\sim}{x}) & \approx x_{2} \frac{\partial \bar{\phi}}{\partial x_{2}}\left({\underset{\sim}{m}}_{m}\right)+ \\
& +\frac{1}{2}\left(x_{\alpha}-x_{m \alpha}\right)\left(x_{B}-x_{m \beta}\right) \frac{\partial^{2} \bar{\phi}}{\partial x_{B} \partial x_{\alpha}}\left({\underset{\sim}{m}}_{m}\right)
\end{aligned}
$$


correct to an error (due to the field scattered by the boss in $\left.S_{m}\right)$ which is $0\left[(a / \ell)^{2}\right]$ smaller.

Summing these results over all area elements $s_{m}$, as previously, combining with (2.16) and substituting into (2.6) we obtain the coherent field representation:

$$
\begin{aligned}
\bar{\phi}(\underset{\sim}{x}) & =\phi_{I}(\underset{\sim}{x})+\phi_{R}(\underset{\sim}{x})+\oint_{S}\left\{\sigma a \frac{\partial^{2} \bar{\phi}}{\partial y_{\alpha}^{2}}-h \frac{\partial^{2} \bar{\phi}}{\partial y_{\alpha}^{2}}\right. \\
& \left.-\left(h-\frac{2 \sigma}{3} a\right) k_{0}^{2} \bar{\phi}-n(1+\sigma) \bar{\phi}\right\} G(\underset{\sim}{x}, L) d y_{1} d y_{3}
\end{aligned}
$$

In other words, for the coherent component, the effective value of $\partial \bar{\phi} / \partial x_{2}$ on $x_{2}=h$ is given by

$$
\frac{\partial \bar{\phi}}{\partial x_{2}}=-(h-\sigma a) \frac{\partial^{2} \bar{\phi}}{\partial x_{\alpha}^{2}}-\left\{\left(h-\frac{2 \sigma a}{3}\right) k_{0}^{2}+n(1+\sigma)\right\} \bar{\phi} \quad,
$$

But $\bar{\phi}$ satisfies the Helmholtz equation (2.1), so that this result is equivalent to

$$
\begin{gathered}
\frac{\partial \bar{\phi}}{\partial x_{2}}=(h-\sigma a) \frac{\partial^{2} \bar{\phi}}{\partial x_{2}}-\left\{n(1+\sigma)+\frac{\sigma a}{3} k_{0}^{2}\right\} \bar{\phi} \\
\left(x_{2}=h\right) .
\end{gathered}
$$

The first term on the right of (2.25) may be discarded, since it accounts for a small uniform phase shift in the reflected field which is of no practical significance. To see this, note that, if the wall were rigid and perfectly flat $(n=0, \sigma=0),(2.25)$ would reduce to

$$
\frac{\partial \bar{\phi}}{\partial x_{2}}-h \frac{\partial^{2} \bar{\phi}}{\partial x_{2}}=0 \text { on } x_{2}=h \text {, }
$$

which, correct to a relative error $\sim 0\left(\mathrm{k}_{\mathrm{O}} \mathrm{h}\right)^{2}$, is the same as the exact rigid surface condition 


$$
\frac{\partial \bar{\phi}}{\partial x_{2}}=0 \quad \text { on } \quad x_{2}=0
$$

In the presence of surface irregularities (2.25) indicates that the effective location of the smoothed plane for application of the boundary condition is $x_{2}=\sigma a$. This is very much smaller than the acoustic wavelength of interest, and henceforth the condition will be applied on $x_{2}=0$, in which case (2.25) reduces to

$$
\frac{\partial \bar{\phi}}{\partial x_{2}}=-i k_{0} n * \bar{\phi}, \quad x_{2}=0
$$

where

$$
n *=\frac{l+\sigma}{Z}-i v k_{o} a
$$

and $v=\sigma / 3$

In obtaining this result it has been implicitly assumed that the hemispherical bosses are sufficiently far apart that mutual interactions via the incoherent field are negligible, an approximation which requires $\sigma<<1$. For finite $\sigma$ the analysis may be modified (along the lines described in Biot's [28] original work, or as described in reference [32]) to deduce that (2.27), (2.28) remain valid provided that the definition of $v$ is modified as follows

$$
\nu=\sigma\left\{\frac{1}{\left(1+\frac{a \sigma}{2 \ell}\right)}-\frac{2}{3}\right\},
$$

where $\ell$ is the minimum distance between the centers of the bosses on $x_{2}=0$. 


\section{One Dimensional Surface Roughness}

For an impedance surface which is irregular in, say, the $\mathrm{x}_{1}$-direction alone, the roughness elements may be modeled by a surface distribution of cylinders ( $N$ per unit length in the $\mathrm{x}_{1}$ - direction) of semi-circular cross-section and radius a. The long wavelength, coherent field boundary condition in this case becomes

$$
\frac{\partial \bar{\phi}}{\partial x_{2}}=-i k_{0} n \star \bar{\phi}-\frac{\pi \sigma}{4} a \frac{\partial^{2} \bar{\phi}}{\partial x_{3}^{2}}, x_{2}=0,
$$

where $\sigma=2 \mathrm{Na}$ is the mean fractional area of the plane $\mathrm{x}_{2}=0$ covered by the cylinders, and

$$
n^{*}=\frac{1+(\pi-1) \sigma}{2}-\frac{i \pi}{4} \sigma k_{0} a \text {. }
$$

These formulae are valid for $\sigma<<1$ only. 


\section{LONG RANGe PROPAGation FROM A POINT SOURCE ClOSE TO THE GROUND: TWO-DIMENSIONAL SURFACE ROUGHNESS.}

The formulae derived above are now applied to study the propagation of low frequency sound over randomly irregular terrain. Both the source and observer are assumed to be located at ground level (or close to he ground, since the wavelength is large). This restriction avoids many of the complications which tend to obscure more general treatments of propagation over an impedance surface.

Consider the outgoing wave solution (for the coherent field) of

$$
\left(\nabla^{2}=k q^{2}\right) \phi=\delta\left(x_{1}\right) \delta\left(x_{2}-\varepsilon\right) \delta\left(x_{3}\right), \quad \varepsilon>0,
$$

where $\phi$ satisfied (2.27) on $\mathrm{x}_{2}=0$, and the overbar on the coherent field is henceforth suppressed. At ground level, $x_{2}=0$, and as $\varepsilon \rightarrow+0$, the following Fourier integral

representation is readily derived with the aid of the boundary conditions

$$
\phi(\rho)=\frac{-1}{2 \pi} \int_{0}^{\infty} \frac{K J_{0}(K \rho) d K}{\left\{\sqrt{K^{2}-k g}-i k_{0} n^{\star}\right\}},
$$

where $J_{0}$ is the zeroth order Bessel function, and $\rho=$ $\sqrt{x_{1}^{2}+x_{3}^{2}}$. A branch cut for $\sqrt{K^{2}-k_{0}}$ in the half-plane $\operatorname{Re} K>0$ extends along the real axis from $k=k_{0}$ to $+\infty$. The path of integration runs along the lower side of the cut ( $\operatorname{ImK}=-0$ ) where $\sqrt{\mathrm{K}^{2}-\mathrm{kg}}$ is real and positive. This configuration ensures that Im $\sqrt{\left.\mathrm{K}^{2}-\mathrm{k}\right\}}<0$ in the cut half-plane Re $\mathrm{k}>0$, which, for an absorbant, irregular surface $(R>0$ in $(2.4))$, implies that the simple pole of the integrand at 


$$
\begin{aligned}
k=k_{0} & (1-n \star 2)^{1 / 2} \\
& \approx k_{0}\left(1-\frac{1}{2} n^{\star 2}\right) \quad\left(n^{\star 2}<<1\right)
\end{aligned}
$$

does not lie in the cut half-plane.

The decomposition $\mathrm{J}_{0}\left(K_{\rho}\right)=\left(\mathrm{H}_{0}^{\left({ }^{1}\right)}\left(\mathrm{K}_{\rho}\right)+\mathrm{H}_{0}^{\left({ }^{2}\right)}\left(\mathrm{K}_{\rho}\right)\right) / 2$ in terms of Hankel functions $\mathrm{H}_{0}^{(1)}, \mathrm{H}_{0}^{\left({ }^{2}\right)}$ permits $(3.2)$ to be replaced by the sum of three integrals. Two of these, the first involving $\mathrm{H}_{0}^{\left({ }^{1}\right)}(\mathrm{K} \rho)$ and the second $\mathrm{H}_{0}^{\left({ }^{2}\right)}(\mathrm{K} \rho)$, are taken respectively along the positive/negative imaginary axes, and sum to zero ([33]), sec. 9.6.4). The third integral is over the branch cut and reduces to

$$
\phi(\rho)=\frac{-1}{2 \pi \rho}\left\{e^{i k_{0} \rho-n *^{2} k_{0} \rho} \int_{0}^{\infty} \frac{H_{0}^{(l)}\left(k_{0} \rho \sqrt{1+\lambda^{2}}\right) d \lambda}{\lambda^{2}+n^{\star 2}}\right\}
$$

([34], page 736). In this expression the first term in the curly brackets is the potential that would be observed on a perfectly flat, rigid surface. For an observer in the acoustic far field $\left(k_{0} \rho>1\right)$, the value of the remaining integral may be approximated by use of the asymptotic formula

$$
\mathrm{H}_{0}^{(1)}\left(\mathrm{K}_{0} \rho \sqrt{1+\lambda^{2}}\right) \approx \sqrt{\frac{2}{\pi k_{0} \rho}} \frac{e^{i\left\{k_{0} \rho \sqrt{1+\lambda^{2}}-\pi / 4\right\}}}{\left(1+\lambda^{2}\right)^{1 / 4}} .
$$

According to the principle of stationary phase the dominant contribution to the integral is from the region $\lambda \ll 1$, and may be obtained by expanding the integrand about $\lambda=0$. In doing this, however, note that the pole of the integrand can lie arbitrarily close to $\lambda=0$ when the specific impedance $z$ is large. To account for this the full effect of the term $\lambda^{2}+n^{\star 2}$ in the denominator must be retained, leading to

$$
\phi(\rho) \approx \frac{-e^{i k_{0} \rho}}{2 \pi \rho}\left\{1-\frac{2 w e^{i \pi / 4}}{\sqrt{\pi}} \int_{0}^{\infty} \frac{e^{i x^{2} d x}}{x^{2}-i w}\right\},
$$


where, in accord with conventional notation,

$$
w=\frac{i k_{0} \rho n \star 2}{2}
$$

is the (complex) numerical distance between source and observer. Since $n^{* 2}<1,|w|$ can assume all values in the range $0<|w|<\infty$ when $k_{0} \rho$ is large. Finally, a series of standard transformations ([33], Sec. 9) enables (3.6) to be expressed in the form

$$
\phi(\rho)=\frac{-e^{i k_{0} \rho}}{2 \pi \rho} F(w)
$$

where $F(w)$ is defined in terms of the complementary error function by

$$
F(w)=1+i \sqrt{\pi w} e^{-w} \operatorname{erfc}(-i \sqrt{w}) \text {. }
$$

In evaluating this expression we take

$$
\sqrt{w}=|w|^{1 / 2} \exp \left\{\frac{i}{2} \arg w\right\}, \text { where } \frac{\pi}{2}<\arg w<3 \pi / 2 \text {. }
$$

This representation of the far field is consistent with previous studies of propagation over a plane impedance surface (c.f. references cited in the Introduction). The function $F(w)$ (hereafter designated the excess attenuation function) accounts for the influence of the irregular impedance surface on propagation relative to the field $\phi_{0}(\rho)=-e^{i k_{0} \rho} / 2 \pi \rho$ obtaining for a smooth rigid plane. $F(0)=1$, and when $|w| \gg 1$,

$$
\begin{aligned}
F(w) & \approx 2 i \sqrt{\pi w} e^{-w}+\frac{1}{2 w}+\ldots \frac{-\pi}{2}<\arg w<\frac{\pi}{2} ; \\
& \approx \frac{1}{2 w}+\ldots \frac{\pi}{2}<\arg w<\frac{3 \pi}{2} .
\end{aligned}
$$


The first term on the right of (3.10a) represents the so-called surface wave. This is exponentially damped if the boundary resistence $R \neq 0$, although there may exist intervals in $|w|$ wherein $|F|>1$. In the extreme case in which $R=0$

$$
w=\frac{-i k_{0} \rho}{2}\left(\frac{1+\sigma}{X}+v k_{0} a\right)^{2} \text {. }
$$

Since it may always be assumed that $k_{0}$ has a small positive imaginary part (corresponding to the existence of viscous dissipation in the acoustic medium), it follows that $\arg w=-\pi / 2+0$, and therefore that, for $|w| \gg 1$,

$$
\phi(\rho) \approx\left(\frac{k_{0}}{2 \pi \rho}, 1 / 2\left|\frac{1+\sigma}{x}+v k_{0} a\right| e^{i\left\{k_{0} \rho\left(1+\frac{1}{2}\left|n^{*}\right|^{2}\right)-\pi / 4\right\}}+0(1 / w \rho)\right.
$$

Thus, the field at ground level is dominated by the surface wave, which propagates subsonically at the phase velocity $c /\left\{1+\frac{1}{2}\left|n^{*}\right|^{2}\right\}$, and decays like $1 / \sqrt{\rho}$.

For an irregular, rigid surface $(X=\infty)$ these results are equivalent to those obtained by Tolstoy [23], and are in accord with experimental observations of Medwin et al [31]. These experiments confirm the predominance of the surface wave in the far field, and in particular that the amplitude decays like $1 / \sqrt{\rho}$ and is proportional to $\mathrm{k}_{0}^{3 / 2}$ (for $\mathrm{X}=\infty$ ). They also establish the validity of predictions of the coherent wave theory for $k_{0} l<1$. 


\subsection{Propagation Over Randomly Irregular Terrain}

The relative importance of irregularities in ground elevation on the propagation of low frequency sound will now be examined. The dependence of the specific normal impedance $z=R+i x$ on the frequency $F=\omega / 2 \pi \mathrm{Hz}$ is approximated by

$$
\begin{aligned}
& R=1+0.051(k / f)^{3 / 4} \\
& X=0.077(k / f) .73
\end{aligned}
$$

$\kappa$ being the flow resistivity of the ground. A reasonable fit to experimental data given in $[2,3]$ for grass covered ground is obtained when $k=230,000$ MKS units. Observe that $|2|^{-1}<0.1$ for $F<450 \mathrm{~Hz}$, and does not excees 0.175 until $\mathrm{f}>1000 \mathrm{~Hz}$. This may be taken to justify conditions imposed above in (2.5). Evidently, for such frequencies the contributions to $n^{*}$ (Equation (2.28)) from $z$ and the surface irregularities can be of the same order of magnitude.

The variation of excess attenuation, $20 \log _{10}|\mathrm{~F}| \mathrm{dB}$ with absolute numerical distance $|w|$ is illustrated in Figures 2(a) 2 (d) for surface irregularities of different sizes and concentrations for the two frequencies $\mathrm{f}=10,100 \mathrm{~Hz}$ (for which $|z|^{-1}=0.0065,0.035$, respectively). The case $\sigma=0.9096$ corresponds to tightly packed hemispherical bosses. The dashed curves denote the excess attenuation ("ground shadowing") for flat ground $(\sigma=0)$, where ultimately $\phi \sim Z^{2} / k_{0} \rho^{2}$ as $\rho \rightarrow \infty$. For flat, rigid ground, $F \equiv 1$, and this characterizes the "near field" behavior of the curves in the figures for small values of $|w|$. When $k_{0} a$ is large the surface irregularities have a significant effect on propagation, the peaks in the curves corresponding to the local predominance of the damped surface wave term (first term on the right of (3.10a)). In such regions 
the onset of the ground shadow zone is delayed by the surface wave to such an extent that the field exceeds that obtaining for a smooth, rigid plane. At larger distances the surface wave is absorbed by the ground.

\section{2 One-Dimensional Surface Irregularities}

In this case formula (3.9) for the excess attenuation $F(w)$ remains valid provided the numerical distance $w$ is defined by

$$
w=\frac{i k_{0} \rho}{2}\left\{\frac{(1+(\pi-1) \sigma)}{2}-\frac{i \pi \sigma k_{0} a}{4} \cos ^{2} \theta\right\}^{2},
$$

for $\sigma<1$. Here $\theta$ is the angle between the direction of propagation parallel to the ground and the $x_{1}$ axis (the surface corrugations being parallel to the $x_{3}$-direction). The variation of the attenuation with $|w|$ for different propagation directions $\theta$ is illustrated in Figs. $3(a),(b)$ for $\sigma=0.2, k_{0} a=.1$ and for $f=10,100 \mathrm{~Hz}$ respectively. At $\theta=900$ the propagation characteristics reduce to those for smooth ground of specific normal impedance $2 /(1+(\pi-1) \sigma)$. 


\section{CONCLUSION}

The theory of low frequency sound propagation over a statistically homogeneous irregular surface of finite impedance has been developed by extension of the Biot/Tolstoy [23-30] theory of coherent scattering by an array of rigid bosses distributed over a plane. In terrestrial applications, the approximations involved are expected to be adequate at frequencies less than about $1000 \mathrm{~Hz}$. The presence of the surface irregularities is responsible for the generation by a groundbased acoustic source of a surface wave which can be effective in penetrating the ground shadow zone predicted theoretically when the ground is modeled as a smooth, plane impedance surface. 


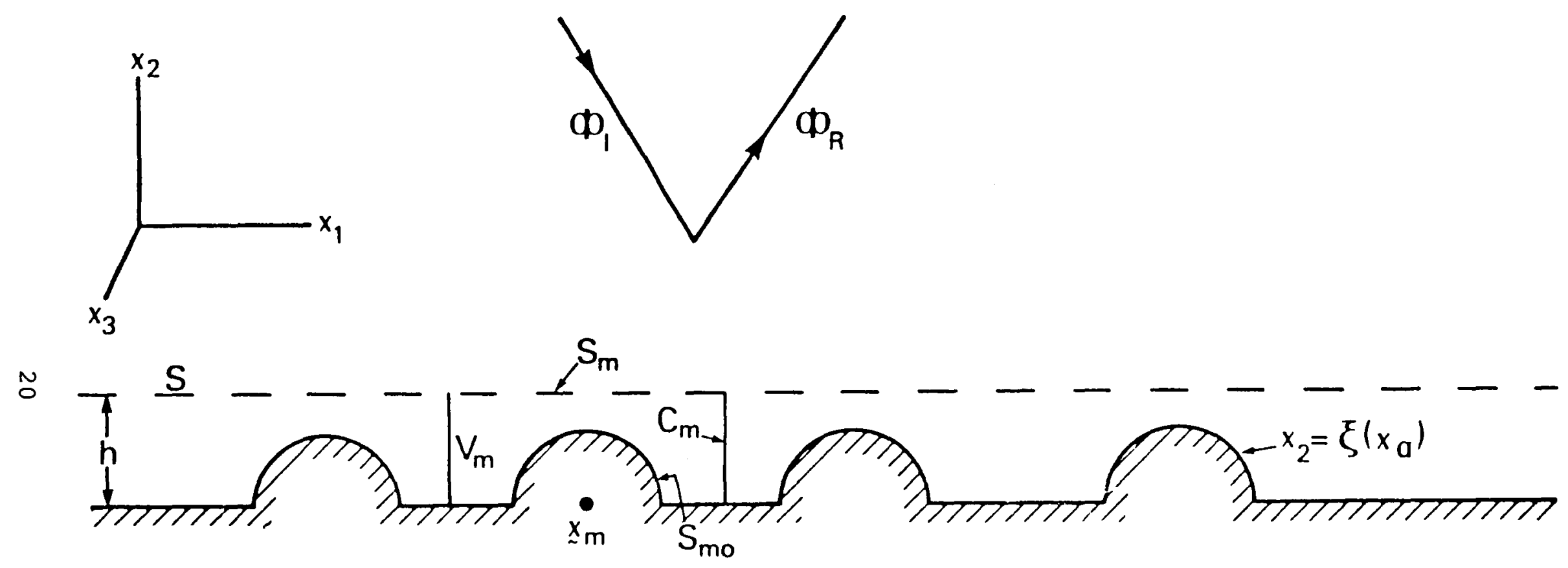

FIGURE 1. Configuration of the idealized randomly irregular impedance surface. 


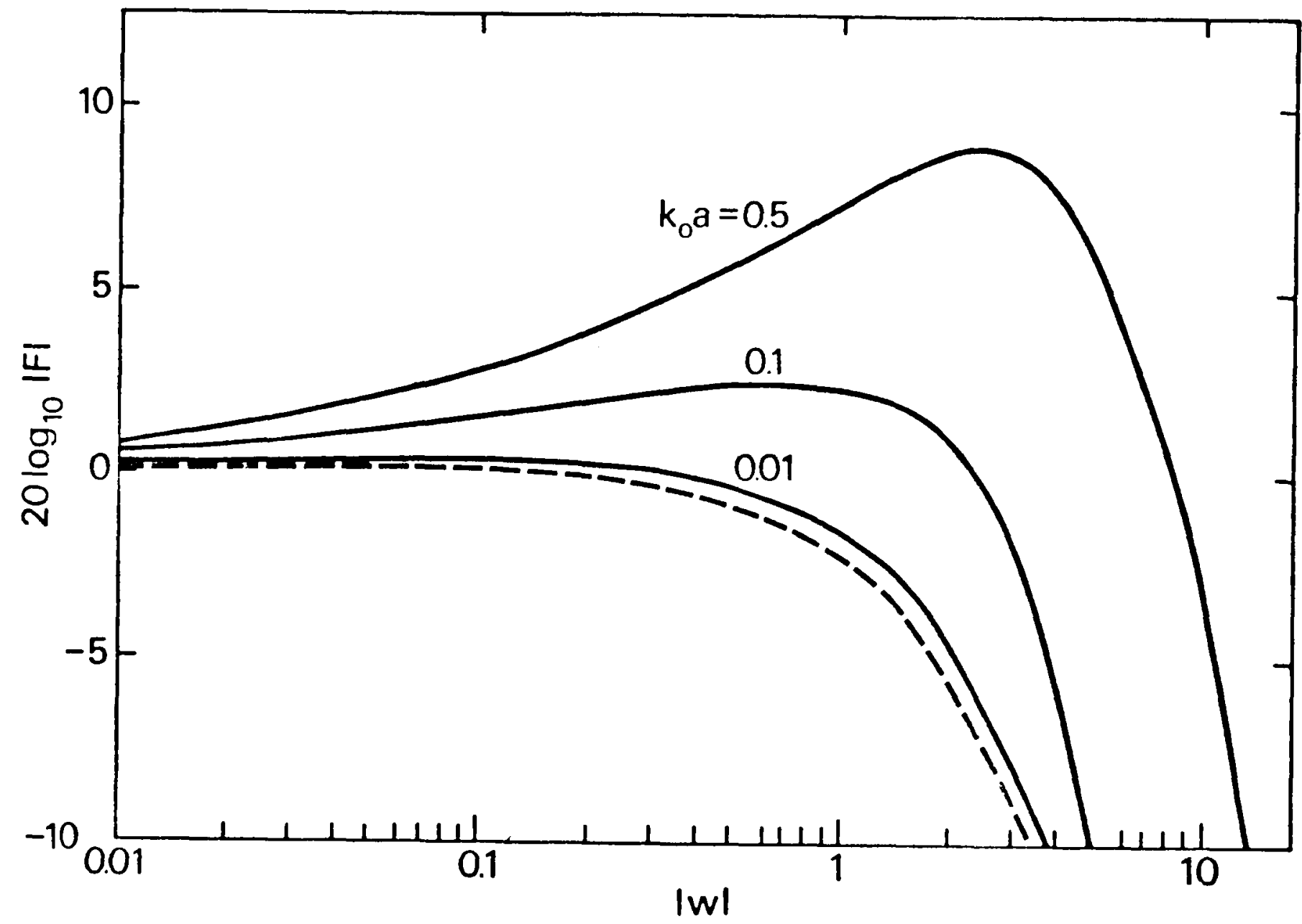

FIGURE 2a. Variation of excess attenuation with absolute numerical distance for irregular terrain of finite impedance modelled by a distribution of hemispherical bosses of radius a.

(a) $\sigma=0.2, \mathrm{f}=10 \mathrm{~Hz}$;

----: excess attenuation for flat ground $(\sigma=0)$ at the same frequency. 


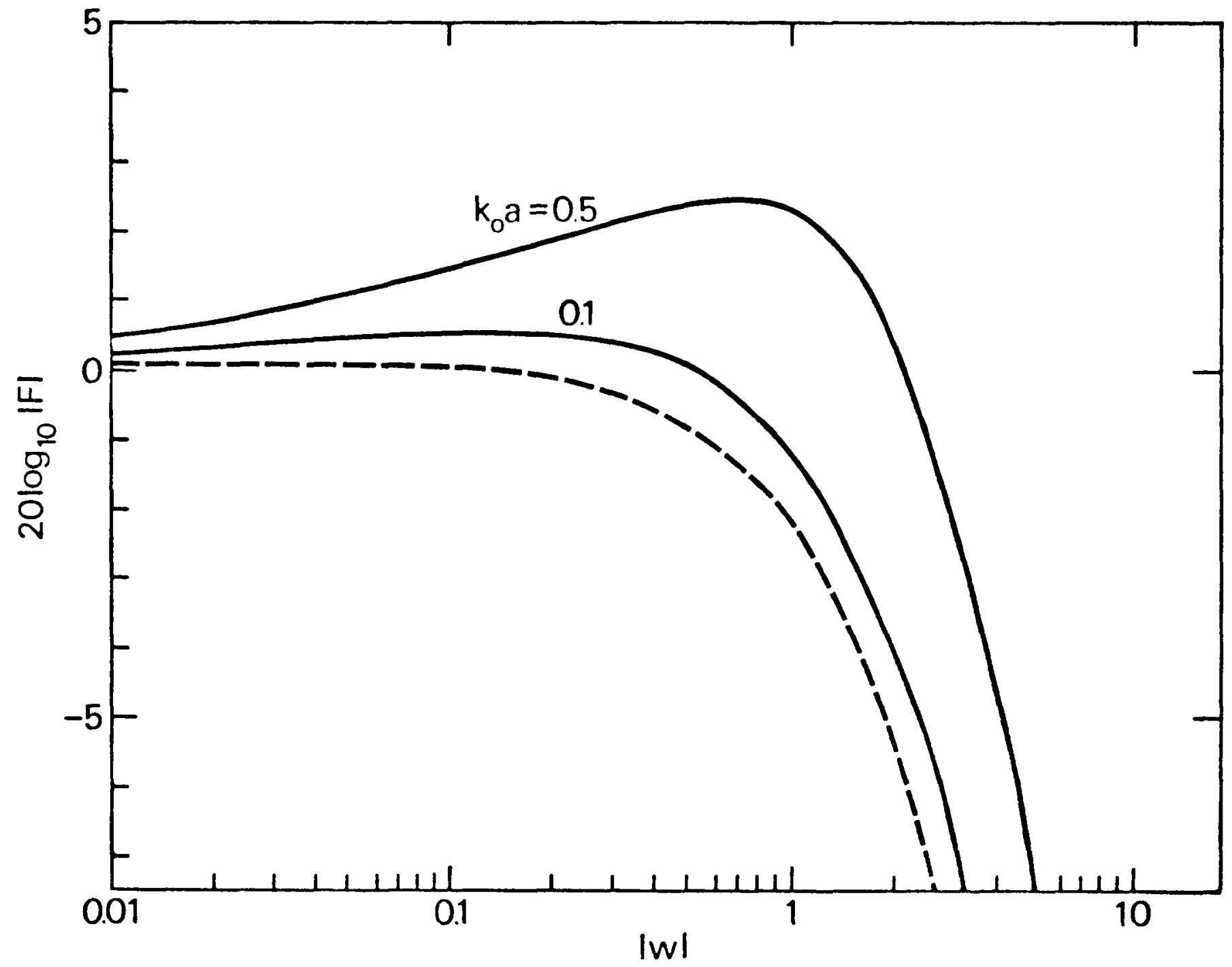

FIGURE 2(b) $\sigma=0.2, f=100 \mathrm{~Hz}$. 


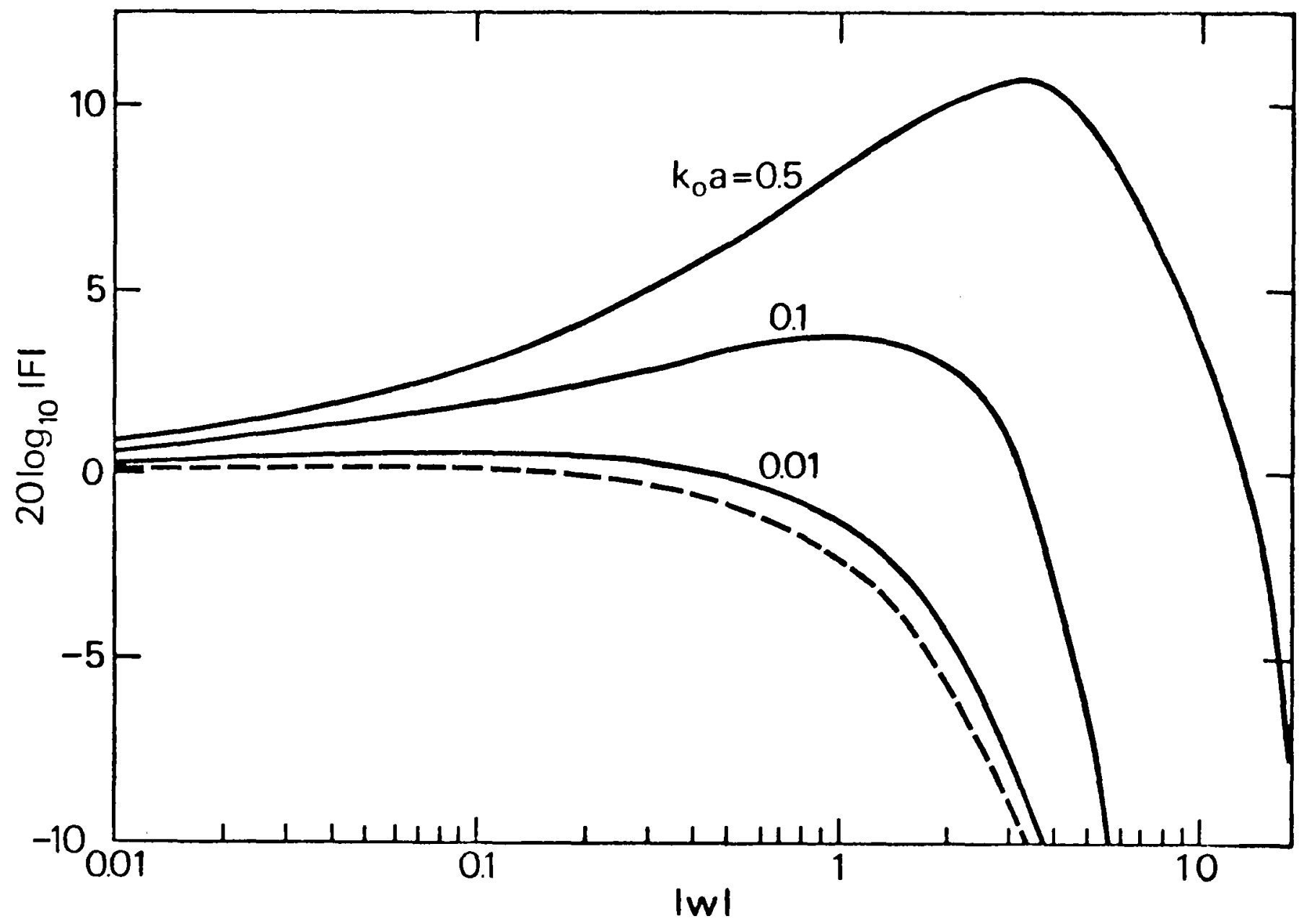

FIGURE 2C $\sigma=0.9096, E=10 \mathrm{~Hz}$; 


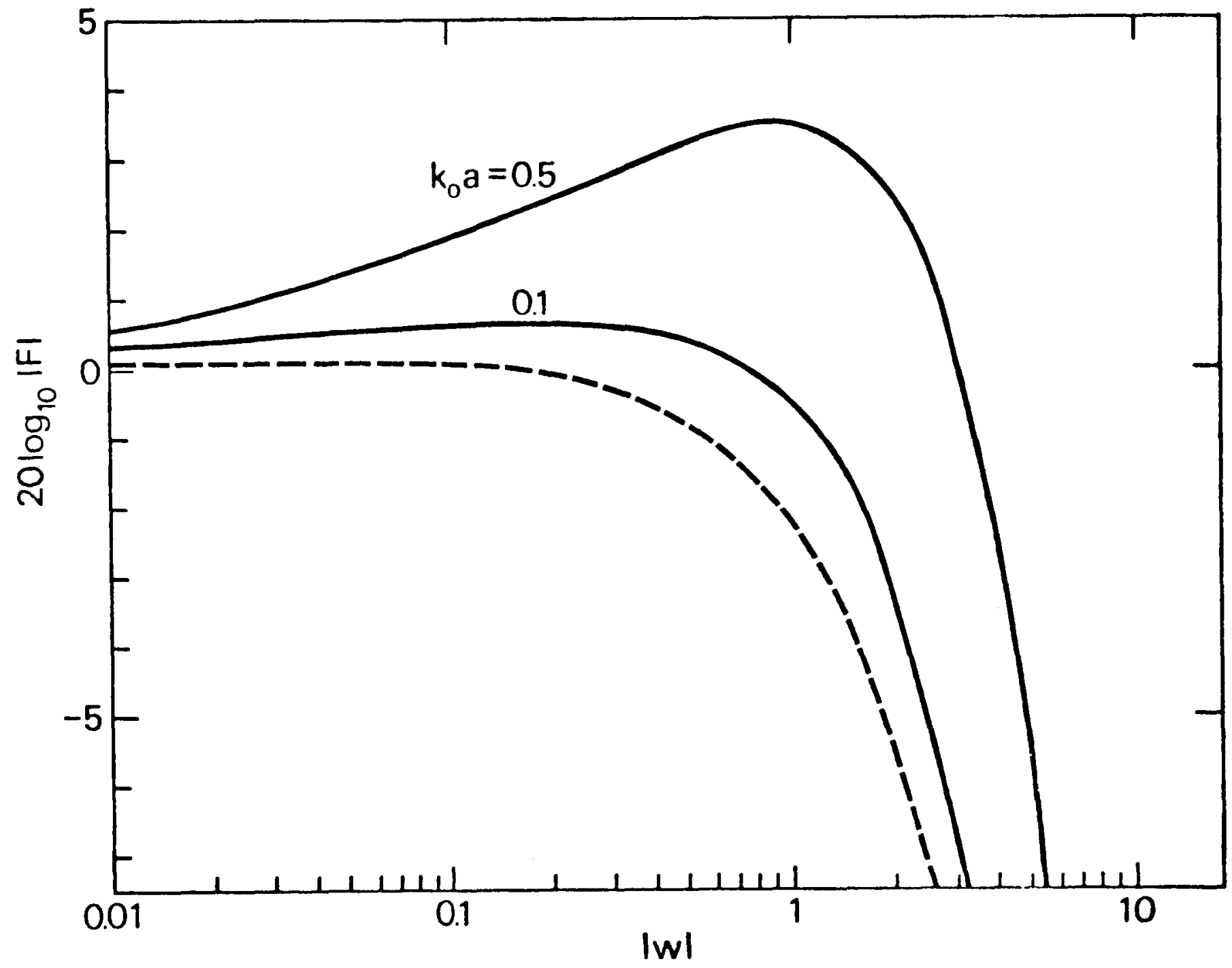

FIGURE 2d. $\sigma=0.9096, E=100 \mathrm{~Hz}$. 


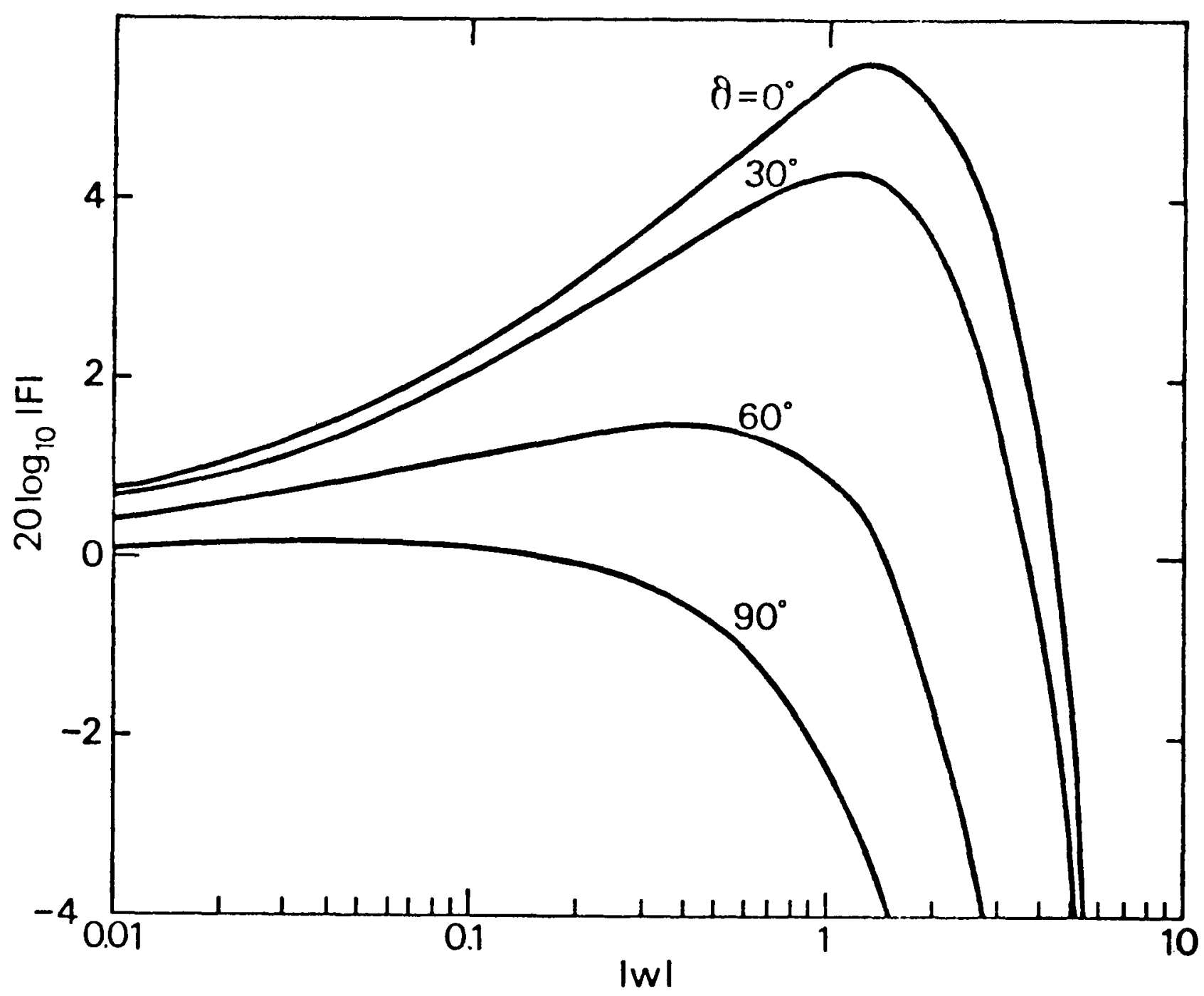

FIGURE 3a. Variation of excess attenuation with absolute numerical distance for irregular terrain of finite impedance modelled by a distribution of parallel cylinders of semi-circular cross-section. $\theta$ is the angle between the propagation direction and the $x_{1}$-axis (normal to the axes of the cylinders); $\sigma=0.2, \mathrm{k}_{0} \mathrm{a}=0.1$ :

(a) $\mathrm{f}=10 \mathrm{~Hz}$; 


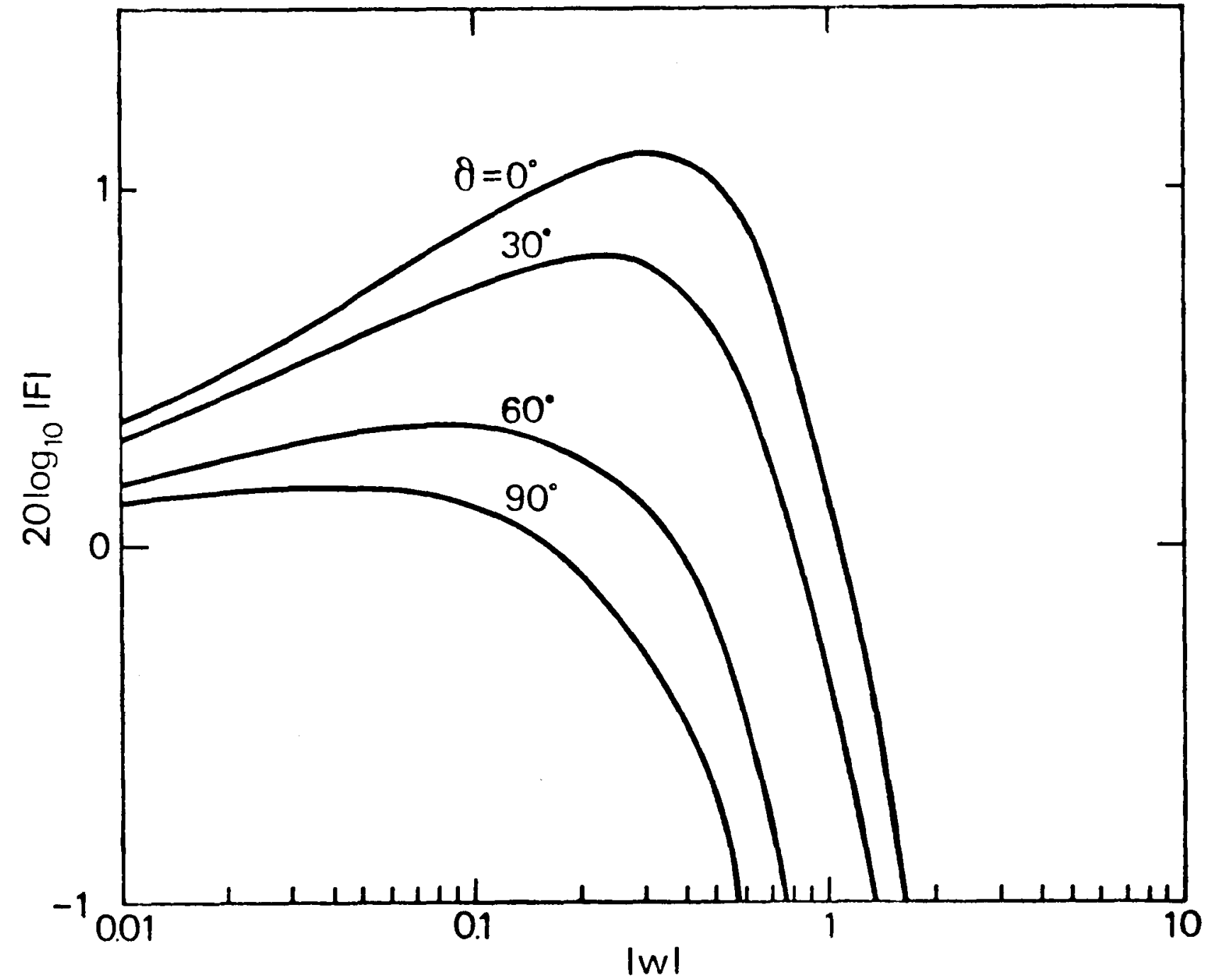

FIGURE $3 \mathrm{~b} . \mathrm{f}=100 \mathrm{~Hz}$. 


\section{$\underline{\text { References }}$}

1. A.D. Pierce, 1981. Acoustics: An Introduction to it's Physical Principles and Applications, New York: McGrawHill.

2. J.E. Piercy, T.F.W. Embleton, and L.C. Sutherland, 1977 . JASA 61:1403-1418. Review of Noise Propagation in the Atmosphere.

3. T.F.W. Embleton, J.E. Piercy, and N. Olson, 1976. JASA, 59:267-277. Outdoor Sound Propagation over Ground of Finite Impedance.

4. S.P. Pao, A.R. Wenzel, and P.B. Oncley, 1978. NASA TP-1104: Prediction of Ground Effects on Aircraft Noise.

5. L.M. Brekhouskikh, 1960, Waves in Layered Media - New York: Academic.

6. P.C. Clemmow, 1966, Plane Wave Spectrum Representation of Electromagnetic Fields. Oxford: Pergammon.

7. J.R. Wait, 1970, Electromagnetic Waves in Stratified Media (2nd Ed.). Oxford: Pergammon.

8. I. Rudnick, 1947. JASA, 19:348-356. Propagation of an Acoustic Wave Along a Boundary.

9. R.B. Lawhead and I. Rudnick, 1951. JASA, 23:546-549. Acoustic Wave Propagation Along a Constant Normal Impedance Boundary.

10. U. Ingard, 1951. JASA, 23:329-335. On the Reflection of a Spherical Sound wave from an Infinite Plane.

11. A.R. Wenzel, 1974. JASA, 55:956-963. Propagation of Waves Along an Impedance Boundary.

12. R.J. Donato, 1976. JASA, 60:34-39. Propagation of a Spherical Wave Near a Plane Boundary with a Complex Impedance.

13. T. Kawai, T. Hidaka, and T. Nakajima, 1982. J. Sound and Vib., 83:125-138. Sound Propagation Above an Impedance Boundary. 
14. K. Attenborough, S.I. Hayek, and J.M. Lawther, 1980. JASA, 68:1493-1501. Propagation of Sound Above a Porous HalfSpace.

15. S.I. Thomasson, 1976. JASA, 59:780-785. Reflection of Waves from a Point Source by an Impedance Boundary.

16. D. Habault and P.J.T. Filippi, 1981. J. Sound and Vib., 79:529-550. Ground Effect Analysis: Surface Wave and Layer Potential Representations.

17. D. Habault, 1981. J. Sound and Vib., 79:551-560. Sound Propagation Over Ground: Analytical Approximations and Experimental Results.

18. T.W. Putnam, 1975. NASA TM X-56033. Review of Aircraft Noise Propagation.

19. C.I. Chesse11, 1977. JASA, 62:825-834. Propagation of Noise Along a Finite Impedance Boundary.

20. K.B. Rasmussen, 1981. J. Sound Vib., 78:247-255. Sound Propagation over Grass-Covered Ground.

21. K. Altenborough, 1982. J. Sound Vib., 81:413-424. Predicted Ground Effect for Highway Noise.

22. R. Raspet, H.E. Bass, and J. Ezell, 1983. JASA, 74:267274. Effect of Finite Ground Impedance on the Propagation of Acoustic Pulses.

23. I. Tolstoy, 1979. JASA, 66:1135-1144. The scattering of Spherical Pulses by Slightly Rough Surfaces.

24. I. Tolstoy, 1981. JASA, 69:1290-1298. Energy Transmission into Shadow Zone by Rough Surface Boundary Wave.

25. I. Tolstoy, 1983. JASA, 73:1192-1199. Coherent Modes and Boundary Waves in a Rough-Walled Acoustic Waveguide.

26. I. Tolstoy, 1982. JASA, 72:960-972. Coherent Sound Scatter from a Rough Interface Between Arbitrary Fluids with Particular Reference to Roughness Element Shapes and Corrugated surfaces.

27. I. Tolstoy, 1982. JASA, 72:973-974. On Scatter from a Rough Interface.

28. M.A. Biot, 1957. JASA, 29:1193-1200. Reflection on a Rough Surface from an Acoustic Point Source. 
29. M.A. Biot, 1958, JASA, 30:479-480. On the Reflection of Acoustic Waves on a Rough Surface.

30. M.A. Biot, 1968, JASA, 44:1616-1622. Generalized Boundary Condition for Multiple scatter in Acoustic Reflection.

31. H. Medwin, J. Bailie, J. Bremhorst, B.J. Savage, and I. Tolstoy, 1979. JASA, 66:1131-1134. On the Scattered Acoustic Boundary Wave Generated by Grazing Incidence at a Slightly Rough Rigid Surface.

32. M.S. Howe, 1984. On the Generation of Sound by Turbulent Boundary Layer Flow Over a Rough Wall. Proc. Roy. Soc. Lond. (in press)

33. M. Abramowitz and I.A. Stegun (eds.), 1964. Handbook of Mathematical Functions. National Bureau of Standards Applied Math. Series No. 55, U.S. Dept. Commerce, Washington, DC.

34. I.S. Gradshteyn and I.M. Ryzhik, 1980. Table of Integrals, Series and Products (corrected edition). New York; Academic. 




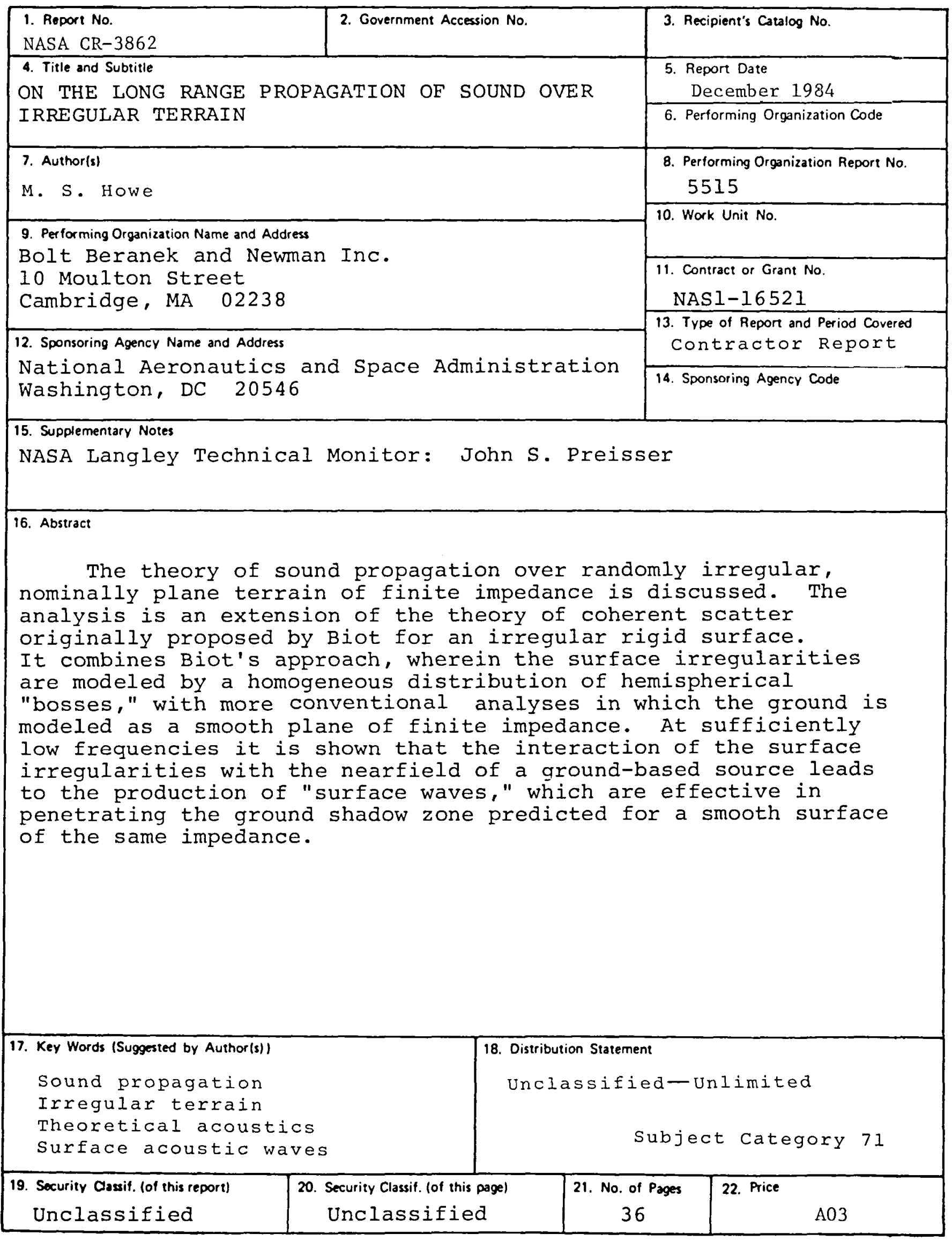

For sale by the National Technical Information Service, Springlield, Virginia 22161 

National Aeronautics and Space Administration

Washington, D.C.

20546

Official Business

Penalty for Private Use, $\$ 300$
Postage and Fees Paid

National Aeronautics and

Space Administration

NASA-451

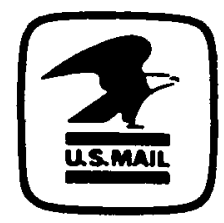

